\title{
Factors associated with breast cancer recurrences or mortality and dynamic prediction of death using history of cancer recurrences: the French E3N cohort
}

\author{
Alexandre Lafourcade ${ }^{1,2}$, Mathilde His ${ }^{3,4}$, Laura Baglietto ${ }^{3,4}$, Marie-Christine Boutron-Ruault ${ }^{3,4}$, Laure Dossus ${ }^{5}$
} and Virginie Rondeau ${ }^{1,2,6^{*}}$

\begin{abstract}
Background: In addition to tumor characteristics and lifestyle factors, cancer relapses are often related to the risk of death but have not been jointly studied. We investigate the prognostic factors of recurrent events and death after a diagnosis of breast cancer and predict individual deaths including a history of recurrences.

Methods: The E3N (Etude Epidémiologique auprès de Femmes de la Mutuelle Générale de l'Education Nationale) study is a prospective cohort study that was initiated in 1990 to investigate factors associated with the most common types of cancer. Overall survival and three types of recurrent events were considered: locoregional recurrence, metastasis, and second primary breast cancer. Recurrent events and death were analyzed using a joint frailty model.

Results: The analysis included 4926 women from the E3N cohort diagnosed with a first primary invasive breast cancer between June 1990 and June 2008; during the follow-up, 1334 cases had a recurrence (median time of follow-up is 7. 2 years) and 469 women died. Cases with high grade, large tumor size, axillary nodal involvement, and negative estrogen and progesterone receptors had a higher risk of recurrence or death. Furthermore, smoking increased the risk of relapse. For cases with a medium risk profile in terms of tumor characteristics and lifestyle factors, the probability of dying between 5 and 10 years after diagnosis was 6,20 and $36 \%$ for 0,1 or 2 recurrences within the first 5 years after diagnosis, respectively.

Conclusions: Our study showed the importance of considering baseline lifestyle characteristics and history of relapses to dynamically predict the risk of death in breast cancer cases. Medical experience coupled with an estimate of a patient's survival probability that considers all available information for this patient would enable physicians to make better informed decisions regarding their actions and thus improve clinical output.
\end{abstract}

Keywords: Breast cancer, Death, E3N cohort, Joint frailty model, Prognostic factors, Recurrences

\section{Background}

Breast cancer is the most common type of cancer in women worldwide and the leading cause of cancer death in women [1]. Between 8 and $10 \%$ of women diagnosed with breast cancer will present locoregional recurrences [2-4], and 15 to $30 \%$ will develop distant metastases [4].

Tumor characteristics are the main prognostic factors for breast cancer outcome. A tumor size larger than $2 \mathrm{~cm}$,

\footnotetext{
* Correspondence: Virginie.Rondeau@inserm.fr

${ }^{1}$ Research Center Inserm, U1219 Bordeaux, France

${ }^{2}$ University of Bordeaux, Bordeaux, France

Full list of author information is available at the end of the article
}

axillary nodal involvement, negative estrogen and progesterone receptors, and high grade have been shown to increase the risk of death after breast cancer diagnosis and the risk of locoregional recurrence and metastases [5]. Young age at diagnosis has also been associated with a worse clinical outcome, partly because of the overrepresentation of more aggressive subtypes in young cases compared to older cases, such as triple negative or HER2positive breast cancer, or because of delayed diagnosis and presentation at an advanced stage [6].

Lifestyle factors might also influence the risk of recurrence and/or death after breast cancer diagnosis. Excess 
weight, which is associated with a higher risk of postmenopausal breast cancer, is also associated with a higher risk of breast cancer recurrence and death [7]. Smoking is weakly associated with breast cancer risk [8] but has been associated with a higher risk of death after breast cancer, with a $60 \%$ higher risk in current smokers at breast cancer diagnosis and a 50\% risk in former smokers of over 35 pack-years $[9,10]$. Similar findings have been reported for the risk of recurrence [10]. Alcohol intake, a risk factor for breast cancer, increases also the risk of recurrence [11, 12]. However studies investigating the interplay between alcohol and other important lifestyle risk factors and certain disease characteristics as well as genetic susceptibility are called for. In contrast, the relation between alcohol consumption and breast cancer survival is less clear [13].

Reproductive factors associated with breast cancer risk could influence breast cancer survival: women with a diagnosis of breast cancer before menopause have a greater risk of recurrence and death [3]. Although hormone replacement therapy (HRT) has been found to increase the risk of breast cancer in healthy women, pre-diagnostic HRT use showed no effect or a decreased mortality in most observational studies [14, 15]. Conversely, the Women Health Initiative (WHI) trial noted that mammary tumors diagnosed in the estrogen plus progestin $(E+P)$ treatment group had worse prognostic characteristics compared to the placebo group [15].

Most studies on prognostic factors for breast cancer recurrence were performed using Cox proportional hazard models or competing-risks regression models [15]. In these studies, the follow-up of the patient ceased after the first relapse, and subsequent recurrences were not considered in the prediction of cancer-related death. The successive relapses can be analyzed using a shared frailty model for the recurrent events to account for the intra-subject correlation. However, in many settings, a joint modeling approach for time to recurrence and for death is required. First, in relation to the recurrences, given the strong relationship between recurrences and breast cancer-related death [3], death should not be considered an independent censoring process. Second, in relation to death, the number of recurrences occurring during follow-up should be considered a timedependent covariate; in this context, the Cox proportional hazards model is not applicable because it requires that the time-dependent covariates be external (or exogenous), that is, their value at a specific time point $t$ is not affected by the occurrence of an event at a previous time point [16].

To overcome these problems, Mauguen et al. [17] proposed to apply a joint frailty model $[18,19]$, that accounted both for the dependence between recurrences and the dependence between recurrences and death.
They showed that when predicting the risk of death, accounting for relapses led to better prediction performance.

In this paper we applied a joint model to study the effects of tumor characteristics, lifestyle characteristics and reproductive factors on the risk of relapse and death after a first invasive breast cancer diagnosis using incident cases within the population-based E3N cohort. More specifically, we studied the dynamic prediction of the risk of death considering baseline lifestyle characteristics and history of recurrent events.

\section{Methods \\ The E3N cohort}

The E3N (Etude Epidémiologique auprès de Femmes de la Mutuelle Générale de l'Education Nationale) study is a prospective cohort study that was initiated in 1990 to investigate factors associated with the most common types of cancer [20]. It includes 98,995 women living in France who were born between 1925 and 1950 and were covered by a national health insurance plan that primarily covers teachers. The participants complete biennial self-administered follow-up questionnaires on health status, medical history and lifestyle. All of the subjects signed an informed consent form at study entry, and the study protocol was approved by the French National Commission for Computed Data and Individual Freedom. The E3N cohort is the French component of the European Prospective Investigation into Cancer and Nutrition (EPIC) and therefore the baseline questionnaire of $\mathrm{E} 3 \mathrm{~N}$ is based on the EPIC questionnaire. Detailed information in English on the EPIC study is available here: http://epic.iarc.fr/index.php.

\section{Ascertainment of recurrent events and death after breast cancer}

The time-to-event was defined as the time between the date of diagnosis of the first invasive breast cancer and the date of each recurrent event or death. Three types of recurrent events were considered: locoregional recurrence, metastasis, and second primary breast cancer. Primary cancers and recurrent events were self-reported to the E3N team by the participants or their next-of-kin or were identified among the causes of death on the death certificate. The information was then validated via pathology reports or any other medical document. We then considered the date of the medical record as the exact date for the first breast cancer diagnosis and for the successive recurrent events. The participants' vital status was regularly updated through the health insurance plan, postal service, and next-of-kin; causes of death were obtained from the French National Service on Causes of Death. In this work we considered all-cause mortality among breast cancer patients. 


\section{Study population}

The study population consisted of 5690 E3N women who were diagnosed with invasive non-metastatic breast cancer confirmed by medical records between June 1990 and June 2008. End of follow-up was defined as the date of death, date of last response to a questionnaire (censored), date of diagnosis of a recurrent event, or June 25, 2008, whichever occurred first. Women with in situ breast cancer $(N=735)$ or a metastatic breast cancer at first diagnosis $(N=29)$ were not included.

Among eligible cases, 242 women declared one or several recurrent events that were not confirmed and were then censored at the date of the event. The type of recurrent event was missing for 9 additional women; these events were removed and the women were censored at the time of the event. Thirty-five women died of breast cancer with metastases reported at death but not before. These events were imputed 2 years before death for 18 women (mean time between all types of relapse and death in this study) and 1 day before death for the others because the delay between cancer and death was less than 2 years.

\section{Statistical analyses}

Recurrent events such as locoregional recurrence, metastasis, or second primary breast cancer may lead to death; conversely, because death prevents any new recurrent events, it is a competing event for recurrences, and these two processes may be correlated. Joint frailty models allow us to study the joint evolution over time of two survival processes by considering all successive recurrent events and the terminal event (death) $[18,19]$. Prognostic factors considered are described in Tables 3 and 4 The characteristics of the first diagnosed tumor were considered: estrogen and progesterone receptor status, differentiation grade, tumor size, axillary nodal involvement. We also considered at the time of diagnosis: age, history of benign breast disease, history of diabetes, history of breast cancer in first-degree relatives, alcohol consumption and Body Mass Index (BMI). Other characteristics included the level of education, smoking status, menopausal status and use of menopausal hormonal treatment (MHT) in the year prior to the breast cancer diagnosis. We also adjusted for the smoking status after diagnosis because women may stop smoking after breast cancer diagnosis. The year of breast cancer diagnosis was used as a proxy for breast cancer care.

We proposed also a dynamic prediction tool to predict the risk of death over a certain period of time, given the history of relapses and the past history in terms of covariates for a patient. The estimated probability can be updated following a new disease relapse. Different scenarios were considered by varying the window of prediction and the number and timing of recurrent events as previously performed in Mauguen et al. [17]. Three different profiles of patients according to risk factors (low, medium and high risk profile) were considered. The analytical framework for the model and this dynamic prediction is provided in the (see Additional file 1). All of the analyses were performed using the Frailtypack 2.6 package of the $\mathrm{R}$ software $[21,22]$.

\section{Results}

During follow-up, among the 4926 cases of the study sample, 549 patients $(11.2 \%)$ were lost to follow-up, 1334 recurrent events occurred including 343 locoregional recurrences, 603 metastases, and $388 \mathrm{~s}$ primary breast cancers; $18 \%$ of women suffered at least one recurrent event, $12 \%$ suffered exactly one event, $4 \%$ suffered two events and 2.1\% suffered three or more) (see Table 1).

The median time between breast cancer and any recurrent event was 7.2 years (IQR: $3.9-11.2$ years). The median time between breast cancer diagnosis and the first recurrence was 3.8 years for the first event, 5.9 years for the second event, and 6.8 years for the third event. Locoregional recurrences generally occurred sooner after breast cancer diagnosis $($ median $=3.9$ years, IQR: $1.2-7.6)$ than metastases $($ median $=4.8$ years, IQR: $2.4-7.9)$ or second primary breast cancers (median $=5.4$ years, IQR: $2.8-8.5$ ). Most events occurring after the first event were metastases (185 subjects, see Table 2).

During follow-up, 469 women died. The median time between breast cancer and death was 5.6 years (IQR: $3.3-8.9$ years). For the women who died, the time between the last non-fatal recurrent event and death decreased with the increasing number of recurrent events. In total, $4.4 \%$ of those with no recurrence, $20 \%$ of those with one recurrence, $50 \%$ of those with two recurrences

Table 1 Statistical summary of number of events: $N=4926$

\begin{tabular}{llll}
\hline & No. & $\%$ & $\begin{array}{l}\text { Time between first } \\
\text { cancer and event }\end{array}$ \\
\cline { 2 - 4 } & & & median [IQR] \\
\hline Number of recurrent events by woman & & \\
0 & 4040 & 82.0 & \\
1 & 604 & 12.3 & $3.8[1.8-7.2]$ \\
2 & 180 & 3.7 & $5.9[3.3-9.2]$ \\
$3-7$ & 102 & 2.1 & $6.8[3.8-9.5]$ \\
Type of event & & & \\
Local recurrence & & & \\
Metastasis & 343 & 25.7 & $3.9[1.2-7.6]$ \\
Second primary breast cancer & 388 & 29.1 & $5.4[2.8-8.5]$ \\
All recurrences & 1334 & 100.0 & $7.2[3.9-11.2]$ \\
Death & 469 & 9.5 & $5.6[3.3-8.9]$ \\
\hline
\end{tabular}

IQR interquartile range 
Table 2 Mean time between two events in years and number of patients observed by type of event, until the 4th event (median follow-up time $=7.2$ years)

\begin{tabular}{|c|c|c|c|c|c|c|c|c|c|c|}
\hline \multirow[b]{2}{*}{ End period event } & \multicolumn{2}{|c|}{ Cancer- 1st event } & \multicolumn{2}{|c|}{ 1st- 2nd event } & \multicolumn{2}{|c|}{ 2nd-3rd event } & \multicolumn{2}{|c|}{3 rd- 4th event } & \multicolumn{2}{|l|}{ TOTAL } \\
\hline & Year $(\sigma)$ & $N(\%)$ & Year $(\sigma)$ & $N(\%)$ & Year $(\sigma)$ & $N(\%)$ & Year $(\sigma)$ & $N(\%)$ & Year (ఠ) & $N(\%)$ \\
\hline \multirow[t]{2}{*}{ Locoregional recurrence } & 4.1 & 276 & 3.4 & 47 & 1.8 & 6 & 2.2 & 7 & 4.9 & 343 \\
\hline & $(3.8)$ & $(5.6)$ & (3.3) & $(5.3)$ & $(2.5)$ & $(2.1)$ & $(2.7)$ & $(7.1)$ & (3.6) & $(5.5)$ \\
\hline \multirow[t]{2}{*}{ Metastasis } & 4.4 & 281 & 1.5 & 185 & 0.9 & 90 & 0.5 & 29 & 2.7 & 603 \\
\hline & (3.5) & $(5.7)$ & $(2.2)$ & $(21.0)$ & $(1.3)$ & $(32.4)$ & $(0.9)$ & $(29.6)$ & $(3.2)$ & (9.6) \\
\hline \multirow[t]{2}{*}{ Second primary breast cancer } & 5.6 & 329 & 3.4 & 50 & 2.6 & 6 & 2.3 & 2 & 5.3 & 388 \\
\hline & $(4.1)$ & (6.6) & (3.4) & $(5.7)$ & (3.6) & $(2.1)$ & (3.1) & (2.0) & (4.0) & (6.2) \\
\hline \multirow[t]{2}{*}{ Death } & 5.3 & 179 & 2.5 & 128 & 1.9 & 91 & 1.3 & 44 & 3.3 & 469 \\
\hline & (3.3) & (3.6) & (2.3) & $(14.6)$ & (1.9) & $(32.9)$ & (1.4) & $(44.9)$ & (3.0) & (7.5) \\
\hline \multirow[t]{2}{*}{ Censored } & 7.4 & 3861 & 5.1 & 464 & 3.3 & 84 & 2.3 & 16 & 7.1 & 4436 \\
\hline & $(4.7)$ & $(78.4)$ & (3.9) & $(53.1)$ & (2.9) & (30.3) & $(2.7)$ & $(16.3)$ & $(4.7)$ & $(71.1)$ \\
\hline \multirow[t]{2}{*}{ TOTAL } & 7.0 & 4926 & 3.8 & 874 & 2.0 & 277 & 1.3 & 98 & 6.1 & 6239 \\
\hline & $(4.7)$ & $(100 \%)$ & (3.6) & $(100 \%)$ & (2.3) & (100\%) & (1.8) & (100\%) & $(4.7)$ & (100\%) \\
\hline
\end{tabular}

$\sigma=$ standard error

and more than $70 \%$ of those with three or more recurrences died during follow-up.

We observed that $13 \%$ of the cases were double hormone receptor negative; $40 \%$ were poorly differentiated; $19 \%$ had a tumor larger than $2 \mathrm{~cm}$ and $28 \%$ had nodal involvement (see Table 3).

We observed that before the cancer diagnosis, $11 \%$ of the women were current smokers (see Table 4). After the cancer diagnosis, only $6 \%$ of the women continued to smoke.

Table 5 presents the results of the multivariate joint frailty model. Negative hormonal receptors, poorly differentiated tumor, large tumor size and nodal involvement were all associated with an increased risk of recurrent events and death. The recent use of MHT was associated with a decreased risk of recurrence (hazard ratio $(\mathrm{HR})=0.75,95 \%$ CI: 0.62, 0.92) but was not associated with the risk of death. Smoking increased the risk of relapse (HR for current smoker vs never smoker $=1.55,95 \%$ CI: $1.16,2.07)$. Breast cancers diagnosed after 2000 were significantly less likely to relapse than those diagnosed before. This result is likely linked to the shorter follow-up time for those with a diagnosis after 2000 and thus a lower probability to observe recurrences. The risk of death was higher when breast cancer was diagnosed after age 60 (HR $=4.50,95 \%$ CI: 1.29, 15.78 for $60-70$ vs $<50$ years and $\mathrm{HR}=9.08,95 \%$ CI: $1.89,43.69$ for $70+$ vs $<50$ years). Menopausal status was not a significant risk predictor.

The risk of death was strongly dependent on the history of recurrence even after adjusting for the covariates (frailty variance $\theta$ and power $\alpha$ significantly different from zero).

\section{Dynamic prediction of the risk of death}

Individual dynamic prediction of death was performed on three profiles of risk factors identified on the basis of
Table 3 Breast cancer characteristics of study population, $N=4926$

\begin{tabular}{|c|c|c|}
\hline & No. of women & Percentage \\
\hline \multicolumn{3}{|c|}{ Estrogen/Progesterone receptors Status } \\
\hline positive / positive & 2321 & 47.1 \\
\hline positive / negative & 770 & 15.6 \\
\hline negative / positive & 161 & 3.2 \\
\hline negative / negative & 636 & 12.9 \\
\hline missing & 1038 & 21.1 \\
\hline \multicolumn{3}{|l|}{ Grade } \\
\hline well differentiated & 650 & 13.2 \\
\hline moderately differentiated & 1517 & 30.8 \\
\hline poorly differentiated & 1965 & 39.9 \\
\hline missing & 794 & 16.1 \\
\hline \multicolumn{3}{|l|}{ Tumor size } \\
\hline$<2 \mathrm{~cm}$ & 3694 & 74.0 \\
\hline$\geq 2 \mathrm{~cm}$ & 956 & 18.5 \\
\hline missing & 276 & 5.6 \\
\hline \multicolumn{3}{|l|}{ Axillary nodal involvement } \\
\hline no & 3233 & 65.6 \\
\hline yes & 1378 & 27.9 \\
\hline missing & 315 & 6.4 \\
\hline \multicolumn{3}{|l|}{ Year of diagnosis } \\
\hline 1990-1994 & 1010 & 20.5 \\
\hline 1995-1999 & 1472 & 29.9 \\
\hline 2000-2003 & 1321 & 26.8 \\
\hline 2004-2008 & 1123 & 22.8 \\
\hline
\end{tabular}


Table 4 Characteristics of the study population, $N=4926$

Age at breast cancer diagnosis
$<50$ years
[50-60) years
[60-70) years
$\geq 70$ years
Menopausal status at breast cancer diagnosis
postmenopausal woman - more than 5 years of
cumulated MHT use
postmenopausal woman - less than 5 years of
cumulated MHT use
postmenopausal woman - no use of MHT
premenopausal woman
Missing
recent MHT use before diagnosis
yes
no
missing
Smoking status before diagnosis
never smoker
current smoker
ex-smoker
missing
Smoking status at first follow-up after diagnosis
never smoker
current smoker
ex-smoker
missing
0

History of benign breast disease before diagnosis

Yes

No

History of diabetes before diagnosis

Yes

No

Level of education before diagnosis

$$
\begin{aligned}
& <\text { high school level } \\
& \text { high school level-second year university level } \\
& >\text { second year university level }
\end{aligned}
$$$$
\text { Missing }
$$

Alcohol consumption at diagnosis

$$
\text { Yes }
$$$$
\text { no }
$$$$
\text { Missing }
$$

BMl at diagnosis

underweight $(<18.5)$

normal $(18.5-<25)$

$\begin{aligned} & \text { No. of } \\ & \text { women }\end{aligned}$ Percentage

$$
\text { women }
$$

647

2145

13.1

1712

43.5

422

34.8

8.6

$1144 \quad 23.2$

1205

1314

1314

923

340

1914

2932

80

2624

562

1717

23

0.5

2624

314

1664

324

3959

804

98

65

2575

2351

258

4668

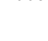

568

2351

1803

204

4105

523

298

132

3255
Table 4 Characteristics of the study population, $N=4926$ (Continued)

\begin{tabular}{|c|c|c|c|c|}
\hline \multirow[t]{2}{*}{ Variables (\% of women) } & \multicolumn{2}{|c|}{ Recurrent event } & \multicolumn{2}{|c|}{ Overall death } \\
\hline & $\mathrm{HR}$ & $95 \% \mathrm{Cl}$ & $\mathrm{HR}$ & $95 \% \mathrm{Cl}$ \\
\hline \multicolumn{5}{|l|}{ Estrogen/Progesterone receptor status } \\
\hline +/- vs +/+ (18.6\%) & 1.40 & $1.10,1.79$ & 2.51 & $1.20,5.30$ \\
\hline$-/+v s+/+(4.1 \%)$ & 1.00 & $0.62,1.59$ & 5.63 & $1.55,20.46$ \\
\hline$-/-v s+/+(15.6 \%)$ & 2.14 & $1.70,2.70$ & 9.76 & $4.39,21.75$ \\
\hline \multicolumn{5}{|l|}{ Grade } \\
\hline $\begin{array}{l}\text { moderately differentiated vs } \\
\text { well differentiated (36.4\%) }\end{array}$ & 1.61 & $1.15,2.27$ & 3.12 & $0.99,9.91$ \\
\hline $\begin{array}{l}\text { poorly differentiated vs well } \\
\text { differentiated }(48.5 \%)\end{array}$ & 1.90 & $1.36,2.66$ & 6.54 & $2.02,21.18$ \\
\hline $\begin{array}{l}\text { Tumor size ( } \geq 2 \mathrm{~cm} \text { vs }<2 \mathrm{~cm}) \\
(20.5 \%)\end{array}$ & 1.64 & $1.33,2.01$ & 4.70 & $2.27,9.75$ \\
\hline $\begin{array}{l}\text { Axillary nodal involvement } \\
\text { (yes vs no) }(30.0 \%)\end{array}$ & 1.54 & $1.26,1.86$ & 5.23 & $2.63,10.42$ \\
\hline $\begin{array}{l}\text { Recent MHT use before diagnosis } \\
\text { (yes vs no) }(44.3 \%)\end{array}$ & 0.75 & $0.62,0.92$ & & \\
\hline \multicolumn{5}{|l|}{$\begin{array}{l}\text { Smoking status first follow-up } \\
\text { after diagnosis }\end{array}$} \\
\hline $\begin{array}{l}\text { ex-smoker vs never smoker } \\
(36.9 \%)\end{array}$ & 1.20 & $1.00,1.44$ & & \\
\hline $\begin{array}{l}\text { current smoker vs never smoker } \\
(6.5 \%)\end{array}$ & 1.55 & $1.16,2.07$ & & \\
\hline \multicolumn{5}{|l|}{ Year of diagnosis } \\
\hline 1995-1999 vs 1990-1994 (28.9\%) & 0.90 & $0.72,1.13$ & & \\
\hline 2000-2003 vs 1990-1994 (32.3\%) & 0.72 & $0.56,0.94$ & & \\
\hline 2004-2008 vs 1990-1994 (27.4\%) & 0.61 & $0.43,0.89$ & & \\
\hline
\end{tabular}

\begin{tabular}{lll}
\hline & $\begin{array}{l}\text { No. of } \\
\text { women }\end{array}$ & Percentage \\
\hline overweight $(25-<30)$ & 1073 & 21.8 \\
obese $(\geq 30)$ & 264 & 5.3 \\
Missing & 202 & 4.1 \\
\hline
\end{tabular}

MHT menopausal hormonal treatment, BMI Body Mass Index

Table 5 Joint model estimation on population ( $N=2708,632$ recurrent events, 212 deaths)

Age at breast cancer diagnosis (year)

$[50-60)$ vs $<50(44.1 \%)$

$1.80 \quad 0.68,4.79$

$[60-70)$ vs $<50(38.0 \%)$

$4.50 \quad 1.29,15.78$

$\geq 70$ vs $<50(9.5 \%)$

$9.08 \quad 1.89,43.69$

Menopausal status at breast cancer diagnosis

postmenopausal woman - no use of MHT vs premenopausal woman (27.1\%)

$1.39 \quad 0.58,3.32$

postmenopausal woman-less than 5 years of cumulated MHT use vs premenopausal woman $(29.3 \%)$

postmenopausal woman-more than

5 years of cumulated MHT use vs premenopausal woman (28.8\%)

$0.79 \quad 0.32,1.97$

$0.35 \quad 0.11,1.10$

$\theta$

$1.01(\sigma=0.05) p$-value $<0.001$

$4.29(\sigma=0.77) p$-value $<0.001$

$H R$ hazard ratio, $\sigma$ standard error, $95 \% \mathrm{Cl}$ 95\% Confidence Interval, $M H T$ menopausal hormonal treatment 
the previous results: a low, medium, high-risk profile (see Fig. 1). When the history of recurrent events was not considered (P2), the probability of death was overestimated if no recurrent events were diagnosed and underestimated if recurrent events occurred.

The number of recurrent events greatly affected the risk of death: for the medium risk profile, the probability of death (P1) between 5 and 10 years after cancer diagnosis was 6\% (95\% CI: 1, 16) for no recurrence in the first 5 years after diagnosis, $19 \%$ (95\% CI: 2, 37) for one recurrence, $36 \%$ (95\% CI: 5, 56) for two recurrences and $53 \%$ (95\% CI: 11, 70) three previous recurrences; between 5 and 15 years, the risk of death was 13\% (95\% CI: 3,28$)$ if no recurrent events occurred and 36\% (95\% CI: 10,57$), 59 \%$ (95\% CI: 23,78$)$ and $76 \%(95 \%$ CI; 38 , 89) if one, two or three events occurred, respectively. The risk of death was also affected by risk factors: after two recurrent events, the risk of death between 5 and 10 years after the cancer diagnosis was 7, 36 and $51 \%$ in women with low-, medium-, and high-risk profiles, respectively.

To estimate whether the predictions are accurate, error of prediction curves based on the brier score were used. Prediction errors (not presented here) were influenced by the number and timing of relapses. Indeed, with a Cox model, the prediction error of the risk of death between 5 and 10 years was $9 \%$, whereas in the joint frailty model, which accounts for relapse times, the prediction error was $8 \%$ with probability P2 and $7 \%$ with probability P1.

\section{Discussion}

In this article, we studied jointly the prognostic factors for successive relapses and death on a large cohort of women with a primary breast cancer. We proposed a dynamic individual prediction of the risk of death after breast cancer using a history of cancer recurrences, tumor characteristics,
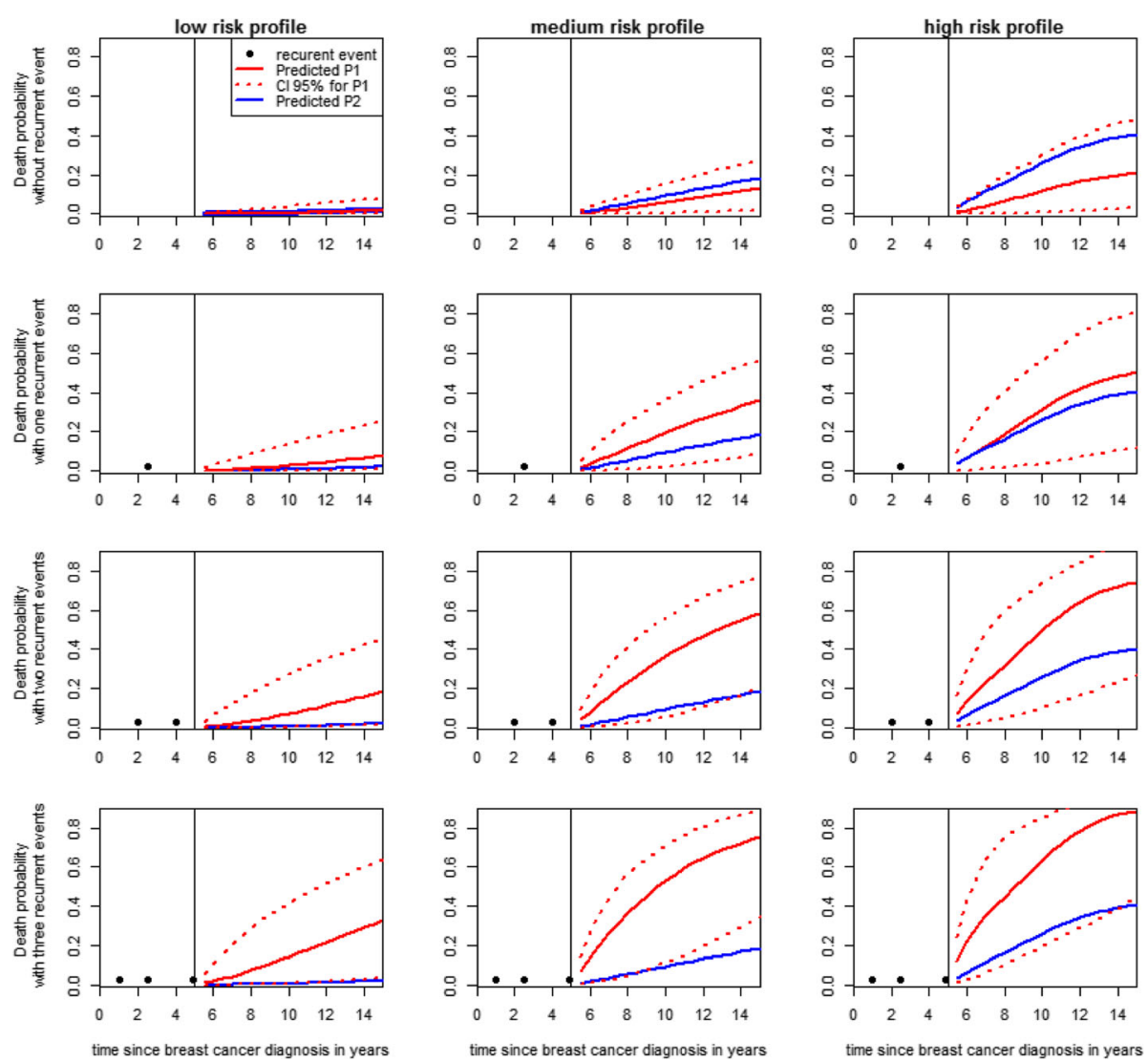

Fig. 1 Individual predicted probabilities of death for 3 patient profiles, with or without a history of relapses using joint frailty models. P1: probability of death considering exactly I recurrent events, $\mathbf{P 2}$ : probability of death not considering the history of recurrent events. Low-risk profile: Estrogen/ Progesterone receptor status $=+/+$, Grade $=1$, Tumor size $\leq 2 \mathrm{~cm}$, Axillary nodal involvement $=$ no, recent MHT use before diagnosis $=$ yes, Smoking status = never smoker, Year of diagnosis = 2004-2008, Age at breast cancer diagnosis $\leq 50$ years, Menopausal status = postmenopausal woman - less than 5 years of cumulated MHT use. Medium-risk profile: Estrogen/Progesterone receptor status $=+/-$, Grade $=2$, Tumor size $\geq 2 \mathrm{~cm}$, Axillary nodal involvement = no, recent MHT use before diagnosis = yes, Smoking status = ex-smoker, Year of diagnosis = 1995-1999, Age at breast cancer diagnosis $=[50-60)$ years, Menopausal status = premenopausal woman. High-risk profile: Estrogen/Progesterone receptor status $=-/-$, Grade $=3-4$, Tumor size $\geq 2 \mathrm{~cm}$, Axillary nodal involvement =yes, recent MHT use before diagnosis=no, Smoking status = smoker, Year of diagnosis = 1990-1994, Age at breast cancer diagnosis $\geq 70$ years, Menopausal status = postmenopausal woman - no use of MHT 
lifestyle characteristics and reproductive factors, showing that at a given time after diagnosis, the risk of death was strongly dependent on the number of relapses.

To date, relatively few studies have attempted to study this association. Our results confirm the relevance of the association between established prognostic factors, MTH and cigarette smoking and the risk of recurrent events or death after breast cancer. The association between lifetime cigarette smoking and risk of breast cancer recurrence has been previously described into the literature [10]. The tumor characteristics (high grade, high tumor size, nodal involvement) were also associated with a higher risk of those two failure times, as previously shown by Mauguen et al. [17]. Conversely, the risk of relapses or death was lower for tumors with positive estrogen or progesterone receptors [23]. We observed as in previous observational studies [24], a lower risk of relapse for postmenopausal women who had used MHT before breast cancer diagnosis. However, women on MHT undergo more frequent mammographic monitoring and thus have an earlier cancer diagnosis [15]. Therefore, the observed better prognosis in terms of relapses for women using MHT in our study is possibly biased. However, we adjusted for the mammographic monitoring as a binary time dependent covariate (with at least one mammographic visit before the time of interest or without mammography). This variable was non-significantly associated with both the risk of recurrence $(\mathrm{HR}=1.25,95 \% \mathrm{CI}: 0.98,1.59)$ and the risk of death $(\mathrm{HR}=0.62,95 \% \mathrm{CI}: 0.27,1.46)$. Furthermore, this adjustment did not change the effect of the MHT use. Menopausal status (with or without history of MHT use) was not significant in the joint frailty model, even if the same tendencies were observed with the use of MHT.

Smoking exposure after breast cancer diagnosis was associated with an increased risk of relapse but not with the risk of death. This might be explained by the fact that almost half of the smokers stopped smoking after their cancer diagnosis. Obesity, a factor that has been previously associated with the risk of recurrence and death after breast cancer [7], was not significant in our study. However, E3N women are relatively slim (less than $6 \%$ were obese at breast cancer diagnosis), which might have resulted in a limited power to detect an association in this subgroup. Our study did not confirm any association of alcohol consumption or education level and the risk of recurrences or death. The year of breast cancer diagnosis was used as a proxy for breast cancer care. We did not obtain a significant association with this year of diagnosis and the risk death however the risk of recurrence was significantly lower after 2000. This might probably be explained by three reasons. Due to the design of the cohort, the breast cancer diagnosed in the 1990'ties will have longer follow-up than breast cancer diagnosed more recently. We also completed these results by a sensitivity analysis on the same sample but more homogeneous in terms of follow-up durations. In this new sample, the follow-up of the subjects was truncated at 10 years and those with a follow-up greater than 10 years were censored at 10 years. The results, on these patients with shorter follow-up times showed really similar findings, with the same association on the risk of recurrence and on death. Furthermore the introduction of Trastuzamab (Herceptin) in 1998 for the adjuvant treatment of Her2+ breast cancer has significantly reduced relapse rates. Finally since 2004 in France, there is a countryside breast cancer screening program for women aged 50-74. This recent screening program is associated with breast cancers diagnosed earlier and at an earlier stage. This can also explain the reduction of recurrence rates with time. However adjusting also for the mammography monitoring as a binary time dependent covariate did not change the associations.

A major limitation of this study is the absence of variables describing the cancer treatments. The year of breast cancer diagnosis, in combination with tumor characteristics, can be viewed as a proxy variable linked to the treatment evolution. The results reflect a lower risk of relapses for those recently diagnosed who are likely using aromatase inhibitors and practicing the technique of sentinel lymph node. However, for patients diagnosed after 1999, the follow-up time is shorter and the probability of observing a relapse is consequently lower.

We have illustrated that some specific profiles of breast cancer patients vary according to tumor characteristics, lifestyle characteristics, reproductive factors and the history of relapses; these factors may predict different probabilities of dying in the future. The proposed approach was able to show that the number of relapses greatly affects the predicted risk of death and that better predictions of death are obtained by considering the history of relapses rather than only considering prognostic factors. For example, the "naïve" probability of death that does not consider the history of relapses was overestimated. Using this individual dynamic prediction tool, we illustrated that death probabilities must be updated each time additional relapses are recorded. In our analysis, we did not consider time-dependent covariates, such as modifications in the patient's treatment, BMI, smoking habits or MHT use. However, similar to how these time-dependent covariates are allowed in the joint modeling, they are allowed in the prediction calculation. Although all of the analyses were performed using a standard statistical R package, a level of expertise in programming may be required. An easy web application as Adjuvant Online [25] could provide the opportunity for every physician to derive updated predictions for new patients when history of relapses or more outcomes (such as longitudinal biomarkers) are available, as in Krol et al. [26]. 


\section{Conclusion}

In conclusion, survival outcomes after a breast cancer are affected by the occurrence of relapses, and the proposed approach (with joint model) is really appropriate to both study their link and to predict the prognosis of patients suffering from a primary breast cancer and with possible relapses. These dynamic prediction tools would be valuable in everyday medical practice. That is, medical experience coupled with an estimate of a patient's survival probability that considers all available information for this patient would enable physicians to make better informed decisions regarding their actions and patient care and thus improve clinical output.

\section{Additional file}

Additional file 1: Joint frailty model and prediction (DOCX $27 \mathrm{~kb}$ )

\section{Abbreviations}

BMI: Body Mass Index; E3N: Etude Epidémiologique auprès de Femmes de la Mutuelle Générale de l'Education Nationale; HER2: Human Epidermal growth factor Receptor 2; HR: Hazard Ratio; HRT: Hormone Replacement Therapy; IQR: Inter Quartile Range; MHT: Menopausal Hormonal Treatment; WHI: Women Health Initiative

\section{Acknowledgements}

The authors are grateful to Rafika Chaït, Lyan Hoang, Laureen Dartois and Celine Kernaleguen for their technical assistance in collecting and cleaning the data. The authors are also indebted to all of the participants for providing the data and to the practitioners for providing the pathology reports.

\section{Funding}

The E3N study is being conducted with financial support from the French League against Cancer, the Mutuelle Générale de l'Education Nationale, the Gustave Roussy Institute, and the National Institute of Health and Medical Research. Laura Baglietto is supported by a Marie Curie International . Mathilde His was supported by a PhD fellowship from the French League against Cancer. This work was partly supported by a grant from INCA (2014-1-PL SHS-03).

\section{Availability of data and materials}

The datasets generated and/or analysed during the current study are not publicly available due ethical reasons, permission by the participants to use their data, according to the signed informed consent, and from the MGEN, their insurance company, is restricted to the team in charge of the cohort, which can be extended to collaborators with a specific research agreement but are available from the corresponding author on reasonable request.

\section{Authors' contributions}

Data analysis and project design: $A L, M H, L D, V R$. Conception and study design: VR, LD, MCBR. Corrections of paper: AL, MH, LB, LD, MCBR, VR. The manuscript has been read and approved for submission by all authors. All the authors have contributed to preparing the manuscript, and no person or persons other than the authors listed have contributed significantly to its preparation.

\section{Ethics approval and consent to participate}

All of the subjects signed an informed consent form at study entry, and the study protocol was approved by the French National Commission for Computed Data and Individual Freedom. E3N was approved by this French Commission for Data Protection and Privacy.

\section{Consent for publication}

Not applicable.

\section{Competing interests}

The authors declare that they have no competing interests.

\section{Publisher's Note}

Springer Nature remains neutral with regard to jurisdictional claims in published maps and institutional affiliations.

\section{Author details}

${ }^{1}$ Research Center Inserm, U1219 Bordeaux, France. ${ }^{2}$ University of Bordeaux, Bordeaux, France. ${ }^{3}$ CESP Generations and Health Team, Paris-Saclay University, Paris-Sud Univ, UVSQ, INSERM, Villejuif, France. ${ }^{4}$ Gustave Roussy, Villejuif, France. ${ }^{5}$ Nutrition and Metabolism Section, International Agency for Research on Cancer, Lyon, France. 'Biostatistic Team, INSERM U1219, University of Bordeaux, 146 rue Léo Saignat, CS 61292, F-33076 Bordeaux Cedex, France.

Received: 2 December 2016 Accepted: 29 January 2018

Published online: 09 February 2018

\section{References}

1. Ferlay J, Soerjomataram I, Dikshit R, et al. Cancer incidence and mortality worldwide : sources, methods and major patterns in GLOBOCAN 2012. Int J Cancer. 2015;136(5):E359.

2. Lowery A, Kell M, Glynn R. Locoregional recurrence after breast cancer surgery : a systematic review by receptor phenotype. Breast Cancer Res Treat. 2012;133(3):831-41.

3. Neri A, Marrelli D, Rossi S. Breast cancer local Recurrence : risk factors and prognostic relevance of early time to recurrence. World J Surg. 2007;31(1):36-45.

4. Voogd AC, Nielsen M, Peterse JL, et al. Differences in risk factors for local and distant recurrence after breast-conserving therapy or mastectomy for stage I and II breast cancer: pooled results of two large European randomized trials. J Clin Oncol. 2001;19(6):1688-97.

5. Soerjomataram I, Louwman MWJ, Ribot JG, et al. An overview of prognostic factors for long-term survivors of breast cancer. Breast Cancer Res Treat. 2008;107(3):309-30.

6. Assi HA, Khoury KE, Dbouk $\mathrm{H}$, et al. Epidemiology and prognosis of breast cancer in young women. J Thorac Dis. 2013;5(Suppl 1):S2.

7. Contiero P, Berrino F, Tagliabue G, et al. Fasting blood glucose and longterm prognosis of non-metastatic breast cancer : a cohort study. Breast Cancer Res Treat. 2013:138(3):951-9.

8. Dossus L, Boutron-Ruault M-C, Kaaks R, et al. Active and passive cigarette smoking and breast cancer risk: results from the EPIC cohort. Int J Cancer. 2014;134(8):1871-88

9. Pierce J, Patterson RE, Senger CM, et al. Lifetime cigarette smoking and breast cancer prognosis in the after breast cancer pooling project. JNCI. 2013;106(1):djt359.

10. Bishop JD, Killelea BK, Chagpar AB, Horowitz NR, Lannin DR. Smoking and Breast Cancer Recurrence after Breast Conservation Therapy. Int J of Breast Cancer. 2014;2014:327081. https://doi.org/10.1155/2014/327081.

11. Holm M, Olsen A, Christensen J. Pre-diagnostic alcohol consumption and breast cancer recurrence and mortality : results from a prospective cohort with a wide range of variation in alcohol intake. Int J Cancer. 2013;132(3):686-94.

12. Kwan ML, Kushi LH, Weltzien E, et al. Alcohol consumption and breast cancer recurrence and survival among women with early-stage breast Cancer : the life after cancer epidemiology study. J Clin Oncol. 2010;28(29):4410-6.

13. Newcomb PA, Kampman E, Trentham-dietz A, et al. Alcohol consumption before and after breast cancer Diagnosis : associations with survival from breast cancer, cardiovascular disease, and other causes. J Clin Oncol. 2013; 31(16):1939-46.

14. Obi N, Heinz J, Seibold P, et al. Relationship between menopausal hormone therapy and mortality after breast cancer the MARIEplus study, a prospective case cohort. Int J Cancer. 2016;138(9):2098-108.

15. Chlebowski RT, Manson JE, Anderson GL, et al. Estrogen plus progestin and breast cancer incidence and mortality in the women's health initiative observational study. J Natl Cancer Inst. 2013;105(8):526-35.

16. Kalbfleisch JD, Prentice RL. The Statistical Analysis of Failure Time Data. Stat. Anal. Fail. Time Data, Second Ed. 2002;95-147.

17. Mauguen A, Rachet B, Mathoulin-Pélissier S, et al. Dynamic prediction of risk of death using history of cancer recurrences in joint frailty models. Stat Med. 2013:32(30):5366-80

18. Rondeau V, Mathoulin-Pelissier $\mathrm{S}$, Jacqmin-Gadda $\mathrm{H}$, et al. Joint frailty models for recurring events and death using maximum penalized likelihood estimation: application on cancer events. Biostatistics. 2007;8(4):708-21. 
19. Rondeau V, Commenges D, Joly P. Maximum penalized likelihood estimation in a gamma-frailty model. Lifetime Data Anal. 2003;9(2):139-53.

20. Clavel-chapelon F, the E3N study group, et al. Int J Epidemiol. 2015;44(3):801-9.

21. Rondeau V, Mazroui Y, Gonzalez JR. FRAILTYPACK: an R package for the analysis of correlated data with frailty models using the penalized likelihood estimation. J Stat Softw. 2012;47(4):1-28.

22. A. Krol, A. Mauguen, Y. Mazroui, A. Laurent, S. Michiels, and V. Rondeau. Tutorial in joint modeling and prediction : A statistical software for correlated longitudinal outcomes, recurrent events and a terminal event. J Stat Softw. 2017;81(3).

23. Dunnwald LK, Rossing MA, Li Cl. Research article hormone receptor status, tumor characteristics, and prognosis : a prospective cohort of breast cancer patients. Breast Cancer Res. 2007;9(1):1-10.

24. Antoine $C$, Liebens F, Carly B, et al. Influence of HRT on prognostic factors for breast cancer : a systematic review after the women ' $s$ health initiative trial. Hum Reprod. 2004;19(3):741-56.

25. Ravdin PM, Siminoff L, Davis GJ, et al. Computer program to assist in making decisions about adjuvant therapy for women with early breast cancer. J Clin Oncol. 2001;19(4):980-91.

26. Krol A, Pignon J, Ducreux M, et al. Joint model for left-censored longitudinal data, recurrent events and terminal Event : predictive abilities of tumor burden for cancer evolution with application to the FFCD 2000 - 05 trial. Biometrics. 2016;72(3):907-16.

\section{Submit your next manuscript to BioMed Central} and we will help you at every step:

- We accept pre-submission inquiries

- Our selector tool helps you to find the most relevant journal

- We provide round the clock customer support

- Convenient online submission

- Thorough peer review

- Inclusion in PubMed and all major indexing services

- Maximum visibility for your research

Submit your manuscript at www.biomedcentral.com/submit 